\title{
Revisiting a longstanding clinical trial exclusion criterion: impact of prior cancer in early-stage lung cancer
}

\author{
Sandi L Pruitt ${ }^{1,2,4}$, Andrew L Laccetti ${ }^{3,4}$, Lei Xuan ${ }^{1}$, Ethan A Halm ${ }^{1,2,3}$ and David E Gerber ${ }^{\star 1,2,3}$ \\ ${ }^{1}$ Department of Clinical Sciences, University of Texas Southwestern Medical Center, Dallas, Texas, USA; ${ }^{2}$ Harold C. Simmons \\ Comprehensive Cancer Center, University of Texas Southwestern Medical Center, Dallas, Texas, USA and ${ }^{3}$ Department of Internal \\ Medicine, University of Texas Southwestern Medical Center, Dallas, Texas, USA
}

Background: Early-stage lung cancer represents a key focus of numerous multicenter clinical trials, but common exclusion criteria such as a prior cancer diagnosis may limit enrollment. We examined the prevalence and prognostic impact of a prior cancer diagnosis among patients with early-stage lung cancer.

Methods: We identified patients $>65$ years of age with early-stage lung cancer diagnosed 1996-2009 in the Surveillance, Epidemiology, and End Results-Medicare linked database. Prior cancers were characterized by type, stage, and timing with respect to the lung cancer diagnosis. All-cause and lung cancer specific-survival rates were compared between patients with and without prior cancer using Cox regression analyses and propensity scores.

Results: Among 42,910 patients with early-stage lung cancer, one-fifth (21\%) had a prior cancer. The most common prior cancers were prostate $(21 \%)$, breast $(18 \%)$, gastrointestinal $(17 \%)$, and other genitourinary $(15 \%)$. Most prior cancers were localized, and $61 \%$ were diagnosed within 5 years of the lung cancer diagnosis. There was no difference in all-cause survival between patients with and without prior cancer (hazard ratio $[H R] 1.01 ; P=0.52$ ). Lung cancer specific survival was improved among patients with prior cancer (HR 0.79; $P<0.001)$.

Conclusions: A prior cancer history may exclude a substantial proportion of patients with early-stage lung cancer from enrollment in clinical trials. Without adverse effect on clinical outcomes, inclusion of patients age $>65$ years with prior cancer in clinical trials should be considered to improve study accrual, completion rates, and generalizability.

Cancer clinical trials are currently in a state of crisis. In the setting of a rapidly advancing portfolio of new therapeutics, our current clinical trial infrastructure is ill-equipped to keep pace (Scoggins and Ramsey, 2010; Kris et al, 2012). Less than five percent of cancer patients in the United States participate in clinical research. Moreover, only 50 percent of National Cancer Institute (NCI)sponsored trials reach completion (Lara et al, 2001; Murthy et al, 2004; Scoggins and Ramsey, 2010). Barriers to patient enrollment are major contributors to delayed or inadequate accrual and include increasingly numerous and complex eligibility criteria (Fuks et al, 1998; Kim et al, 2015). Well-reasoned, comprehensive eligibility criteria are essential to maximizing safety, cross-trial comparison and selection of patients most likely to benefit from experimental treatment. Unfortunately, many eligibility criteria are reflexively incorporated into trial protocols, not because they are scientifically justified, but because they are carried over from

*Correspondence: Dr DE Gerber; E-mail: david.gerber@utsouthwestern.edu

Presented in abstract form at the Annual Meeting of the American Society of Clinical Oncology, Chicago, IL, USA, June 3-7, 2016.

${ }^{4}$ These authors contributed equally to this work.

Received 17 July 2016; revised 1 January 2017; accepted 16 January 2017; published online 14 February 2017

(C) 2017 Cancer Research UK. All rights reserved 0007-0920/17 
previous protocols for similar patient populations (Kim et al, 2015).

Critically reconsidering whether these legacy eligibility criteria make sense is essential to improving the pace and generalizability of cancer research, and to providing fair access to new therapies. This need is particularly pronounced for early-stage lung cancer, for which there is a meaningful likelihood of cure. Timely research into optimal management including surgery, radiation therapy, adjuvant chemotherapy, and other medical treatments such as molecularly targeted drugs and immunotherapy is a priority. Furthermore, the widespread implementation of computed tomography (CT)-based lung cancer screening is likely to result in a nationwide increase in the incidence of early-stage lung cancer, rendering research into these cases more important than ever (Siegel et al, 2015).

Patients with a prior cancer diagnosis are routinely excluded from lung cancer clinical trials and the impact of this practice on study accrual appears substantial. In our prior analysis of Eastern Cooperative Oncology Group (ECOG)-endorsed lung cancer clinical trials, over 80 percent of trials excluded patients with prior cancer, resulting in exclusion of up to $18 \%$ of target accrual (Gerber et al, 2014). Presumably this practice reflects concerns that a prior cancer diagnosis will negatively impact treatment options or prognosis (due to the potential for prior cancer recurrence or adverse effects from prior treatment).

Earlier studies investigating the impact of prior cancer diagnosis on early-stage lung cancer outcomes are small, single-centered, and demonstrate variable results (Liu et al, 2002; Duchateau and Stokkel, 2005; Lopez-Encuentra et al, 2007; Aguilo et al, 2008). A large, nationally representative study of patients with advanced (stage IV) and locally advanced (stage III) lung cancer suggested that the overwhelming majority of prior cancers were in situ or localized stage, and that individuals with a prior cancer diagnosis had outcomes as good as, if not slightly better than, those without prior cancer diagnoses (Laccetti et al, 2015, 2016).

Although intriguing, these findings in advanced and locally advanced disease cannot be extrapolated to early-stage lung cancer, for which aggressive local therapies are pursued and 5-year survival may exceed 70\% (Goldstraw et al, 2007). Accordingly, we determined the prevalence, type, stage, timing, and prognostic impact of prior cancer diagnoses among patients with early-stage lung cancer using a large national dataset.

\section{MATERIALS AND METHODS}

Data Acquisition. This study was approved by the Institutional Review Board of the University of Texas Southwestern Medical Center (IRB\# STU 082012-040). Data were obtained from linked 1992-2009 National Cancer Institute Surveillance, Epidemiology, and End Results (SEER) program data and 1991-2011 Medicare claims data. Ninety-four percent of cancer patients reported to SEER aged 65 years or older have been successfully linked with Medicare data (Warren et al, 2002). Data for this study were available from 17 registries broadly representing $\sim 26 \%$ of the US population (Altekruse et al, 2010).

Study Population. This study included Medicare patients age $>65$ years diagnosed with early-stage primary lung cancer between 1992 and 2009. We defined early stage disease as American Joint Committee on Cancer (AJCC) Stage I and II, per the AJCC Cancer Staging Manual, Third and Sixth Editions (Adamo and Ruhl, 2015). We selected 1992 as the initial year for the study because Medicare claims were first available in 1991, allowing a one-year lead-in time to capture pre-existing comorbidies. We selected 2009 as the latest year for the study because it was the most recent year of available data at the time of analysis.
We included individuals age $>65$ years to allow for one-year of complete Medicare claims data pre-diagnosis to capture preexisting comorbidies. All patients had full coverage of Medicare Parts A and B from one year before and one year after the lung cancer diagnosis. To ensure complete claims data, we excluded HMO members and patients with only autopsy or death certificate records. We included only patients with non-small cell lung cancer (NSCLC) or small cell lung cancer (SCLC) histology. We excluded patients with incomplete diagnosis or death dates or discrepancies in SEER and Medicare birth dates of a year or more.

Measures. The outcomes of interest were all-cause and lung cancerspecific mortality. Survival was measured as the interval in months between diagnosis date (defined as the 15th of the month because SEER provides only month and year of diagnosis) and death date per SEER. Patients were followed until date of death or censored at the end of 2009 (last date of death in 2011 SEER submission).

A history of prior cancer was determined as described in previous studies (Gerber et al, 2014; Laccetti et al, 2015). Briefly, prior cancers were identified using the SEER variable tumor site recode; patients whose first primary cancer was a lung cancer $($ siterkm1 $=39$ ) were defined as not having a prior cancer. Those whose second or subsequent primary cancer was a lung cancer (siterkm2-10=39) were defined as having a prior cancer. We measured characteristics of prior cancers including cancer type, stage, and timing in relation to the index lung cancer. For patients with more than one primary lung cancer, the first primary lung cancer was considered the index lung cancer. We did not consider these patients to have a prior cancer history because (1) it is challenging to accurately identify same-site second primary cancers using registry data (Coyte et al, 2014) and (2) in clinical practice it may be difficult to distinguish between a second primary lung cancer versus recurrent disease.

We examined multiple covariates previously shown to be associated with lung cancer prognosis including treatments such as surgery within 120 days of diagnosis, chemotherapy within 120 days of diagnosis, and radiotherapy within one year of diagnosis (Warren et al, 2008; Ahn et al, 2013; Laccetti et al, 2015). ICD-9 and CPT codes used to identify these measures have been described previously (Warren et al, 2008; Laccetti et al, 2015). We measured comorbidity by searching inpatient, outpatient, or carrier claims for multiple chronic conditions within 12 months pre-diagnosis using the Charlson comorbidity index-Klabunde adaptation (Charlson et al, 1987; National-Cancer-Institute, 2016). Patients with Medicaid were identified using the state buy-in variable (Koroukian et al, 2010).

Statistical Analysis. Using descriptive statistics, we reported the prevalence and correlates of a prior cancer diagnosis, including the type and stage of prior cancer, and the time interval between the prior cancer diagnosis and index lung cancer diagnosis. We used unadjusted Kaplan-Meier analysis to compare survival functions for patients with and without a prior cancer history for both outcomes. Kaplan-Meier curves were also constructed according to characteristics of the most recent prior cancer diagnosis, including timing of diagnosis (within $\leqslant 1$ year; $\leqslant 3$ years; $\leqslant 5$ years of the index lung cancer), cancer stage, and cancer type.

Three separate Cox proportional hazard models were employed for each outcome: univariable, multivariable and propensity scoreadjusted models. The multivariable model included all measured sociodemographic, cancer type, comorbidity, and treatment covariates shown in Table 1. We further fitted a propensity-score adjusted model and compared findings to the multivariable model. Propensity scores were constructed to adjust for observable differences (i.e., potential confounders) between patients with and without a prior cancer diagnosis using a logistic model containing all measured covariates. We examined propensity score overlap and balance across covariates using multiple regression, chi-square 
Table 1. Baseline patient characteristics of the early stage lung cancer SEER-Medicare cohort $(N=42,910)$

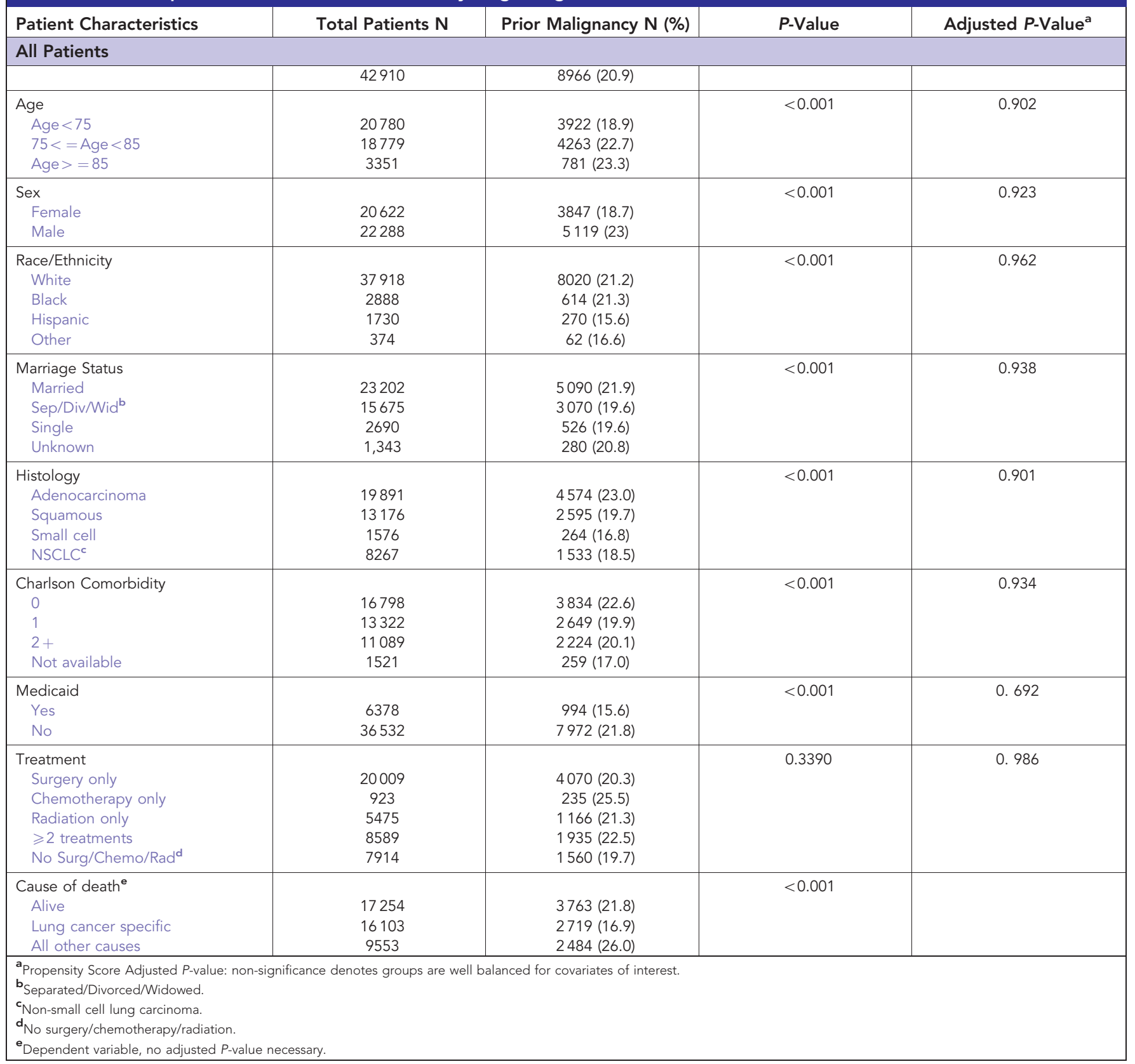

analysis, and histograms. Cox models were fitted adjusting for propensity scores as a continuous variable.

To confirm the validity of our results, we conducted two sensitivity analyses. First, we performed a subset analysis to better represent a clinical trial-eligible population. In this multivariable covariate-adjusted Cox model, we included only patients who received surgery as treatment of the index early stage lung cancer. Second, we conducted a sensitivity analysis to account for potential lead time. It is possible that, patients with a prior cancer could be receiving closer monitoring, more imaging, and a faster work-up of symptoms, thus, earlier diagnosis of lung cancer than otherwise would be expected. A diagnosis earlier in the natural history of the disease could result in lead time bias. To account for this potential bias, our sensitivity analysis employed an approach previously used in studies of cancer screening and surveillance testing (Friedman and Dubin, 1991; Schootman et al, 2008). Specifically, we conducted a matched case-control analysis where cases had died (analyzed separately by all-cause and lung-cancer specific death) and controls were alive at the end of follow-up. Cases and controls were matched on survival time in one-month increments, thus accounting for potential lead time, and the association of prior cancer with death was examined using logistic regression. We applied Coarsened Exact Matching (CEM), which offers several advantages over traditional matching methods, and is described in detail elsewhere (Iacus et al, 2011, 2012). We fitted unadjusted and multivariable covariate-adjusted logistic regression models. Analyses were performed using SAS 9.3 (SAS Institute Inc. Cary, NC, 2013) and Stata 13.1 (StataCorp. LP., College Station, TX, 2013).

\section{RESULTS}

We identified 42910 patients with early-stage lung cancer. Among these cases, one-fifth $(21 \%, N=8966)$ had a history of prior cancer. Forty-eight percent of patients were less than 75 years of age, 52\% were male, and $47 \%$ of patients underwent surgical resection alone. Additional characteristics are displayed in Table 1. 
A Type of most recent prior cancer

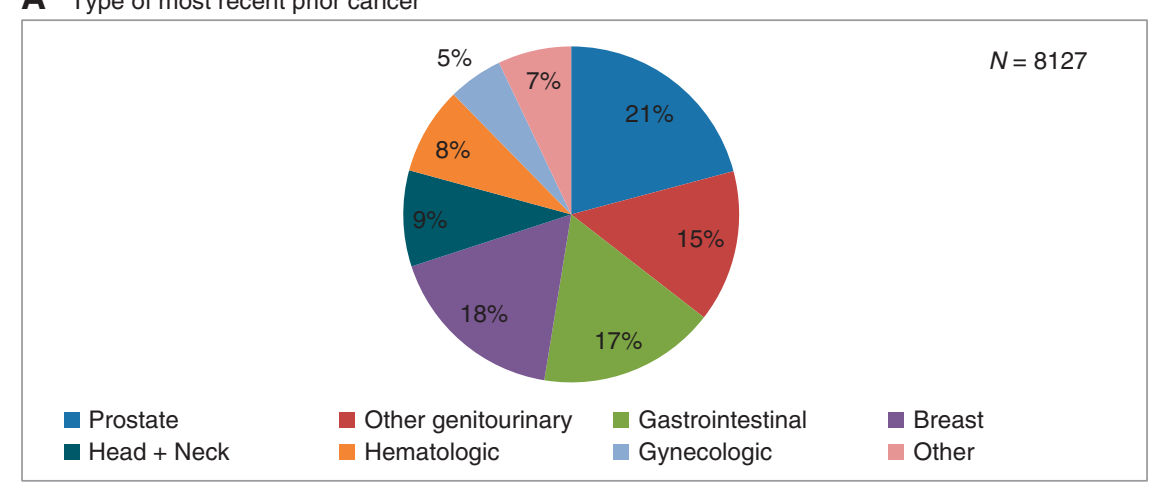

B Stage of most recent prior cancer

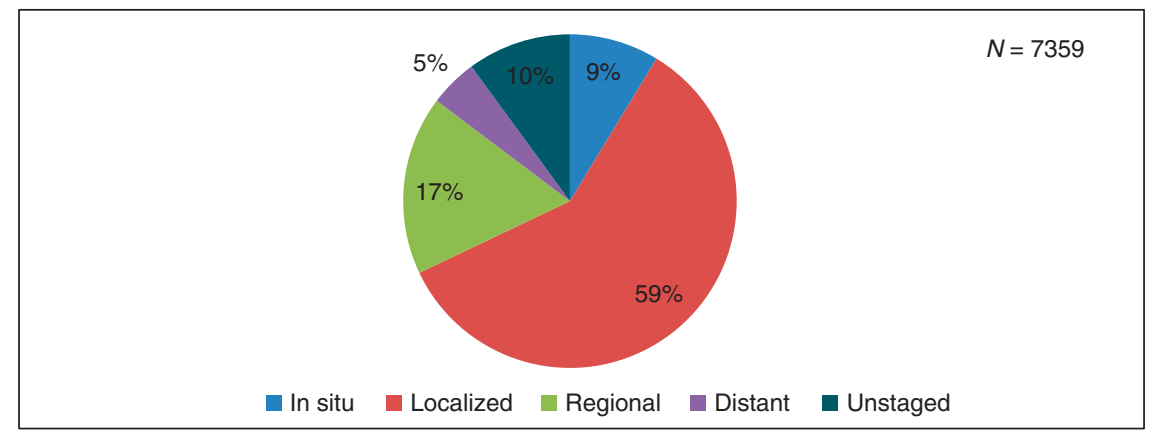

C Timing of most recent prior cancer

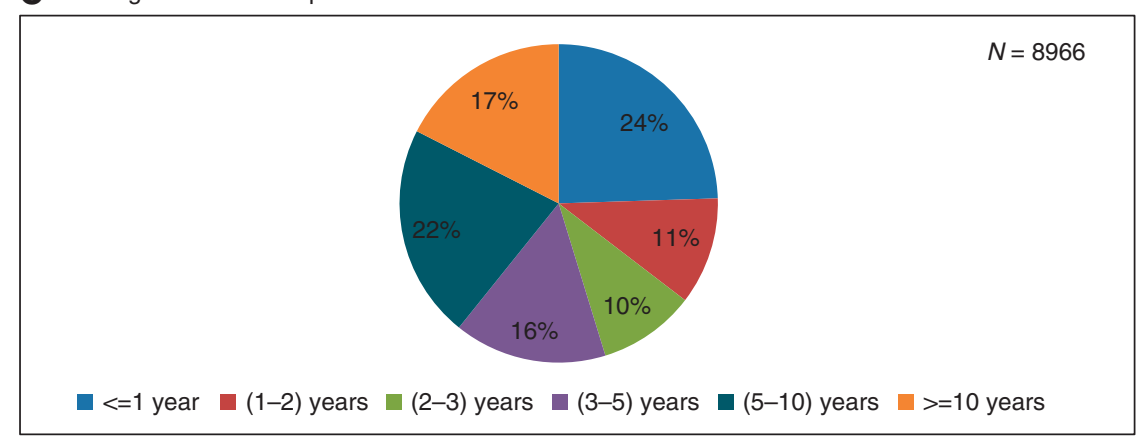

Figure 1. Type (A), stage $(\mathbf{B})$ and timing $(\mathbf{C})$ of the most recent prior cancers. (Note: Cell sizes $<11$ are suppressed per the SEER-Medicare data use agreement; Denominators are not equal due to missing data).

Figure 1 depicts the type (a), stage (b) and timing (c) of prior cancer diagnoses. The most common types of prior cancer cancers were prostate $(21 \%)$, breast (18\%), gastrointestinal (17\%) and other genitourinary (15\%). Sixty-eight percent of prior cancers were either in situ or localized stage, while only $5 \%$ were distant stage. The median time between the most recent prior cancer diagnosis and the index lung cancer diagnosis was 3.6 years (mean 5.3 years, standard deviation [SD] 6.0 years). Having multiple prior cancer diagnoses was infrequent: only $3 \%$ of patients (1315) had two prior cancers, and $0.5 \%$ (197) had three prior cancers. Among these multiple prior cancer cases, prior cancer type and stage distribution were similar to single prior cancer cases (data not shown). Median/ mean time between the prior cancer diagnosis and the index lung cancer diagnosis were 7.0/8.9 years (SD 7 years) for the second most recent prior cancer diagnosis and 7.9/12.0 years (SD 8.3 years) for the third most recent prior cancer diagnosis.

In unadjusted Kaplain-Meier analysis, early-stage lung cancer patients with a prior cancer demonstrated significantly different all-cause and lung-cancer specific survival (log rank tests $P<0.05$ ), compared to patients without a prior cancer (Figure 2). While curves visually overlap for all-cause survival, a clear survival advantage is visible for lung-cause specific survival. Figure 3 depicts all-cause Kaplain-Meier survival curves stratified by the timing, stage, and type of the most recent prior cancer. For analyses by prior cancer timing, although $P$ values are significant or marginally significant for each comparison, the survival curves visually overlap and there is not a clinically meaningful difference (Figure 3A). Patients with in situ, localized and regional stage prior cancers demonstrated non-inferior survival compared to patients with no prior cancer, whereas patients with unstaged or distant stage prior cancer had inferior survival, compared to patients without prior cancer. By type of prior cancer, surival was best among those with a history of breast cancer. Patients with a prior prostate, other genitourinary, or gastrointerstinal cancer had outcomes similar to those of patients without prior cancer.

Results from the unadjusted Cox proportional hazard models, multivariable covariate-adjusted models, and propensity-score adjusted models were similar in effect size, direction, and significance. We present the multilvariable covariate-adjusted models (Table 2). For all-cause mortality, patients with a prior cancer had equivalent all-cause mortality (hazard ratio [HR]: 1.01; 95\% CI: $0.98-1.04)$ but decreased lung-cancer specific mortality 
A

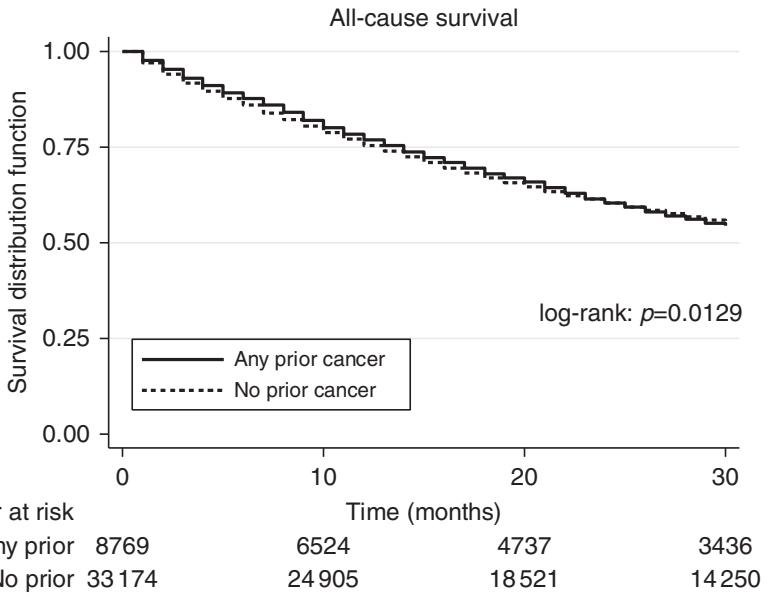

B

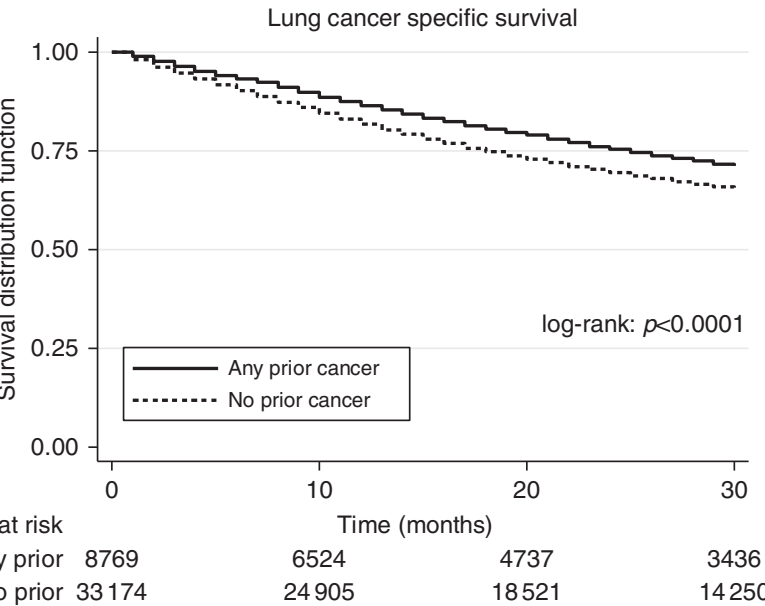

Figure 2. All-cause (A) and lung cancer-specific (B) survival for patients with and without prior cancer.

(HR: 0.79 ; 95\% CI: 0.76-0.82), compared to patients without a prior cancer.

To approximate a population that might be considered for early-stage lung cancer clinical trials, we analyzed the subset of patients who underwent surgical resection of the index lung cancer $(N=25596)$, which represented $60 \%$ of the total study cohort (data not shown). Within this subset, one-fifth $(21 \%, N=5,436)$ also had a prior cancer diagnosis. In a covariate adjusted multivariable model, all-cause survival was slightly worse among patients with prior cancer (HR 1.13; 95\% CI, 1.08-1.18; $P<0.001$ ), whereas lung cancer-specific survival was slightly better among patients with prior cancer (HR 0.91; 95\% CI, 0.85-0.96; $P<0.001$ ).

In our sensitivity analyses designed to account for lead time bias (data not shown), we observed the following. For both outcomes, both unadjusted (all $P_{s}<0.001$ ) and multivariable covariateadjusted models (all Ps $<0.001$ ) demonstrated that patients with prior cancer were less likely to die, compared to patietns without prior cancer. Thus, lead time bias did not account for results of our primary analysis demonstrating improved (lung-cancer specific) or equivalent (all-cause) survival for patients with prior cancer.

\section{DISCUSSION}

Rational clinical trial design, including well-reasoned selection of inclusion and exclusion criteria, is essential for efficient, equitable, and generalizable cancer research. The American Society of Clinical Oncology and the National Cancer Institute have both identified strategies for augmented clinical trial accrural as vital areas of investigation (Scoggins and Ramsey, 2010; Denicoff et al, 2013). This need is particularly evident for early-stage lung cancer. Compared to other early-stage common solid tumors, such as breast, prostate, and colorectal, survival for stage I-II lung cancer is relatively poor. Despite a clear mandate for therapeutic innovation in this population, it is estimated that only $8 \%$ of stage I and $20 \%$ of stage II lung cancer patients are eligible for clinical trials, with medical comorbidities and functional status being the primary reasons for exclusion (Horn et al, 2013). Given the anticipated substantial increase in the numbers of these cases due to uptake of computed tomography-based lung cancer screening, pragmatic and innovative clinical trials are needed more than ever.

In this nationally representative analysis of Medicare patients with early-stage lung cancer, we found that one-fifth of patients had at least one prior cancer diagnosis. This suggests that the common practice of excluding patients with a prior cancer diagnosis from clinical trials in early-stage lung cancer may substantially limit trial accrual. This proportion exceeds those among patients with either locally advanced (15\%) or metastatic (16\%) lung cancer (Gerber et al, 2014; Laccetti et al, 2015), and is also greater than the prior cancer rate of $13 \%$ described among all cancer types (Levi et al, 2014). A potential explanation is that, in some cases, the prior cancer diagnosis and ensuing clinical and radiographic follow-up lead to an early-stage lung cancer diagnosis in an otherwise asymptomatic individual. Notably, given the fourfold increase in the number of cancer survivors in the U.S. over the past 30 years (de Moor et al, 2013), the frequency of prior cancers is likely to increase in the future. 
A
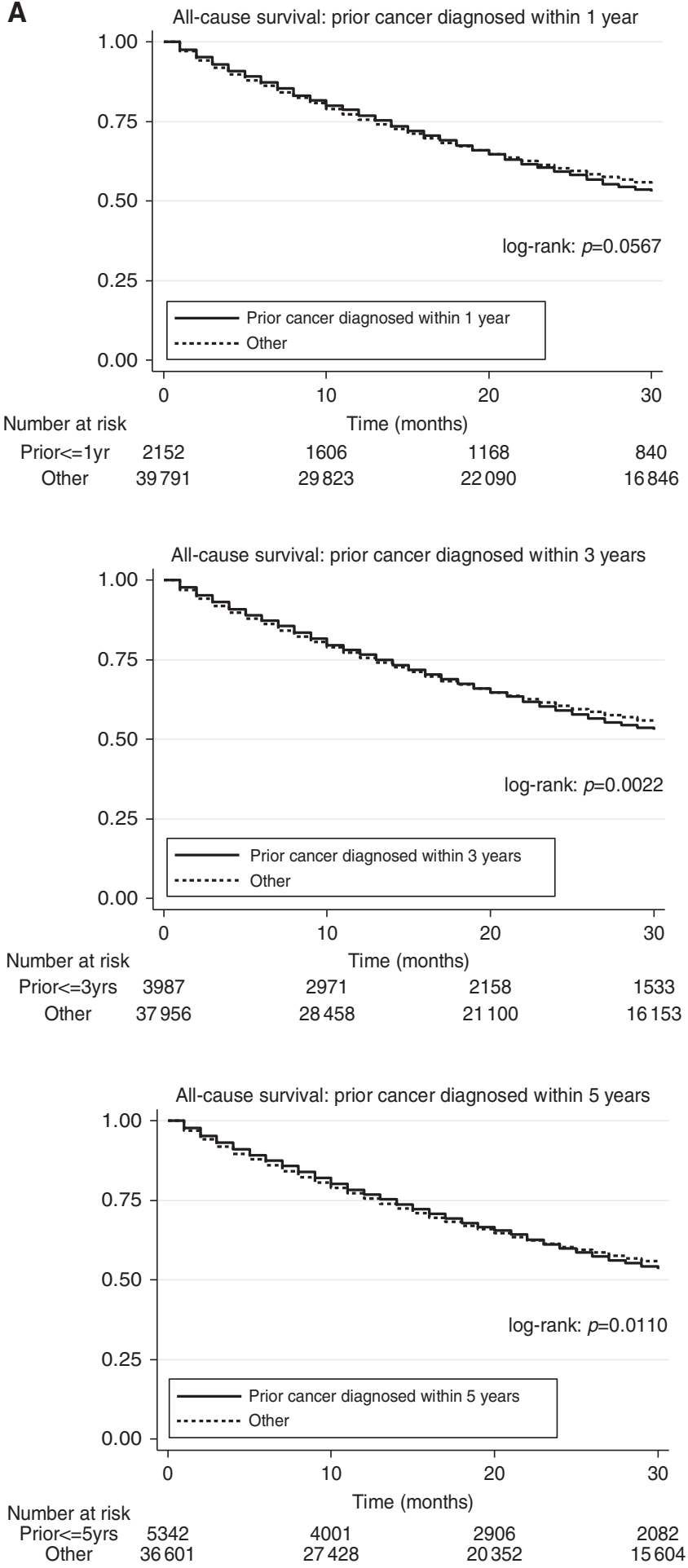

B All-cause survival by prior cancer stage

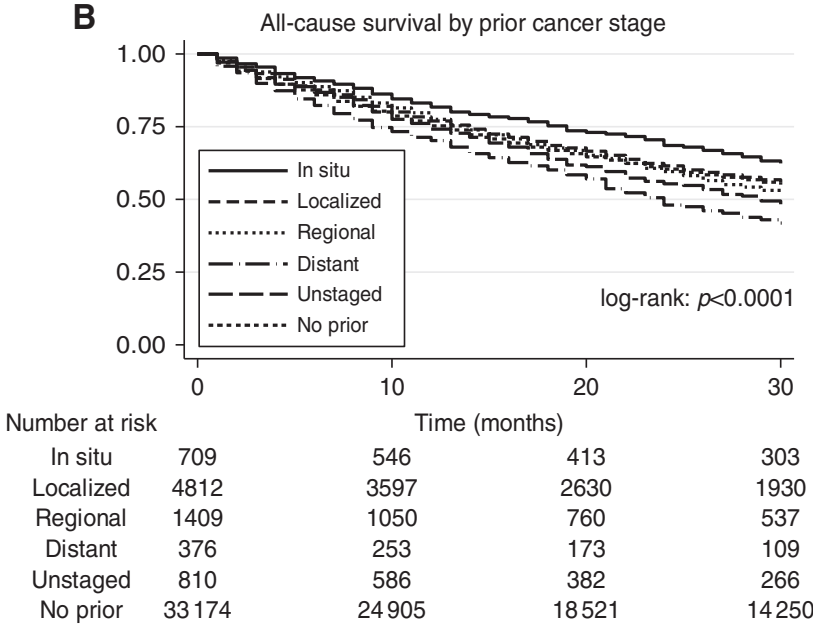

C

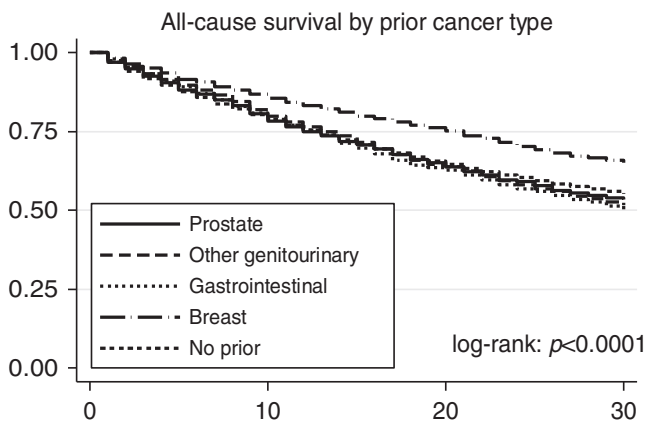

Number at risk

Prostate $\quad 1839$

Other genitourinary 1274

Gastrointestinal 1498

$\begin{array}{cc}\text { Breast } & 1528 \\ \text { No prior } & 33174\end{array}$

33174

Figure 3. All-cause survival according to timing (A), stage (B), and type (C) of prior cancer diagnosis ('Other' denotes patients with no prior malignancy or a history of prior malignancy diagnosed outside the referenced time frame).

If individuals with prior cancer diagnoses are excluded from early-stage lung cancer clinical trials due to perceived risk that they will not tolerate aggressive lung cancer treatment or will have inferior outcomes, our findings may alleviate some of this concern. Rates of surgical and multi-modality treatment were simliar between patients with and without a prior cancer diagnosis. Furthermore, the majority of prior cancer diagnoses had highly favorable characteristics. Almost two-thirds were in situ or localized stage. Prostate, other genitourinary (primarily bladder), and breast cancer-which are often diagnosed at early stage, potentially indolent, and associated with excellent prognosisaccounted for almost $45 \%$ of all prior cancers. Our adjusted mortality analyses found that a history of prior cancer did not adversely effect outcomes. All-cause mortality was similar between patients with and without prior cancer diagnoses $(\mathrm{HR}=1.01)$. Adjusted lung cancer-specific mortality was actually better among those patients with prior cancer diagnoses $(\mathrm{HR}=0.79)$.

The observation that patients with a history of prior cancer may have better lung cancer-specific survival is consistent with our prior SEER-Medicare studies of patients with metastatic and locally 
Table 2. Multivariable covariate-adjusted hazard ratios for all cause and lung cancer specific mortality

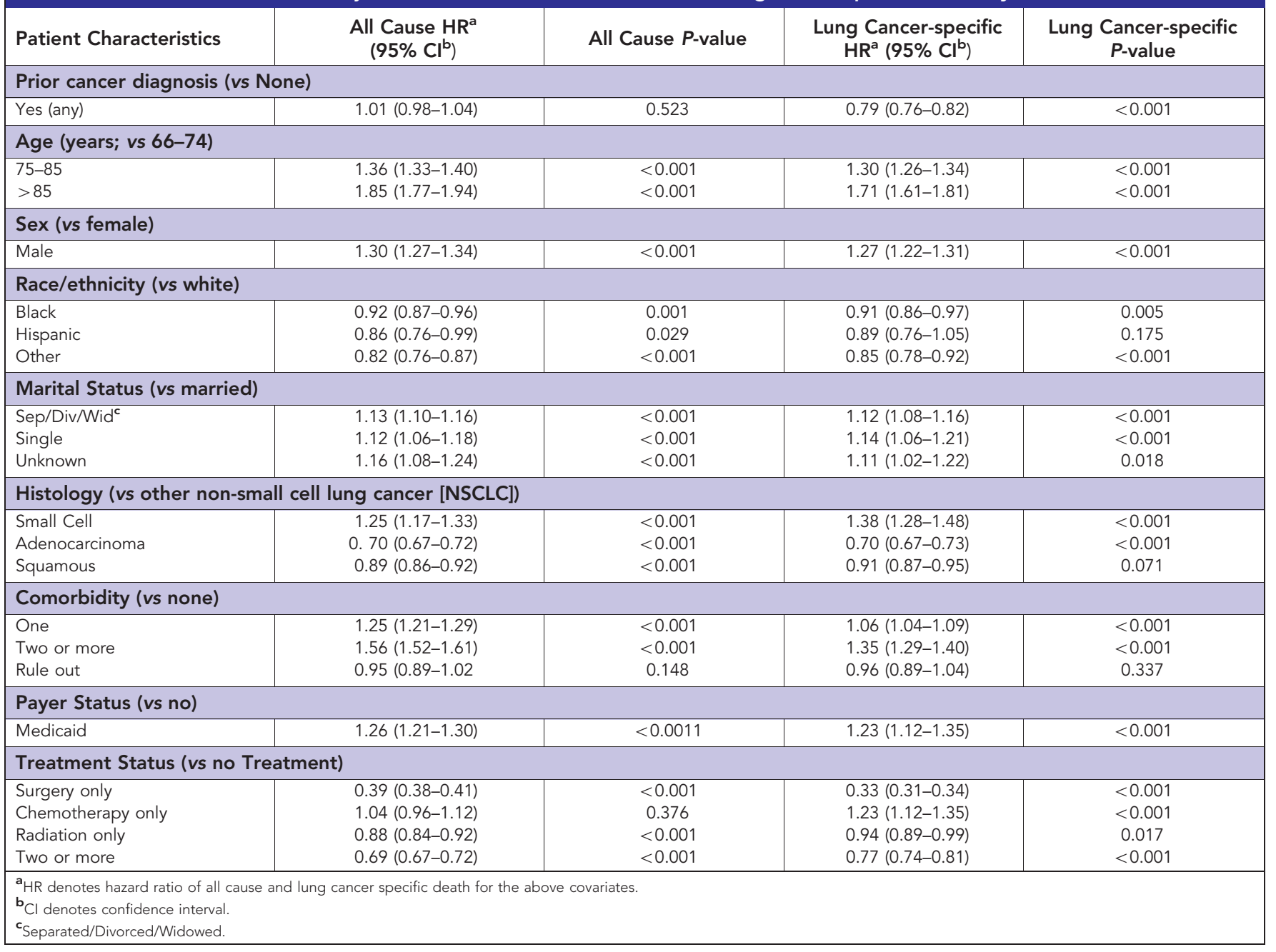

advanced lung cancer (Laccetti et al, 2015, 2016). Earlier singlecenter studies have also reported improved outcomes for those lung cancer patients with a history of prior cancer (Liu et al, 2002; Duchateau and Stokkel, 2005; Aguilo et al, 2008). Potential explanations for this finding include an advantageous cancer survivor phenotype or lead-time bias (Sankila and Hakulinen, 1998; Gerber et al, 2014). That is, patients undergoing routine follow-up for a previous cancer diagnosis may have their earlystage lung cancer diagnosed at an earlier point in its clinical history compared to patients whose early-stage lung cancer comes to clinical attention for other reasons. Our sensitivity analyses suggested lead time bias was not responsible for the lung cancerspecific survival advantage observed in this study. It should be noted, however, that results of these analyses are not directly comparable to the overall analysis (because different statistical models are used for each). Finally, that we did not observe an improvement in all-cause survival among patients with prior cancer may reflect competing risks, either due to the earlier cancer or other variables.

Characteristics of a prior cancer diagnosis may inform clinical trial eligibility considerations. Importantly, the time-frame of the prior cancer diagnosis in relation to the index lung cancer diagnosis did not meaningfully impact survival trends, suggesting that patients with relatively short intervals between the two cancer diagnoses could be considered for enrollment in early-stage lung cancer trials. This observation is highly relevant because (1) most lung cancer clinical trials mandate at least a 5 -year interval between a prior cancer diagnosis and enrollment (Gerber et al, 2014), and
(2) most prior cancer diagnoses occur within 5 years of the lung cancer diagnosis. The particularly favorable outcomes for patients with prior breast cancer compared to patients with other prior cancers and patients with no prior cancer may represent a gender effect. These patients are overwhelmingly female, and women have superior lung cancer outcomes compared to men (Fu et al, 2005; Wisnivesky and Halm, 2007; Sagerup et al, 2011; Tong et al, 2014).

Patients with a history of an advanced stage (metastatic) cancer ( $5 \%$ of our cohort) had worse outcomes compared to patients with no prior cancer diagnosis. Almost universally, these patients harbor an incurable malignancy with relatively poor prognosis, with perhaps the single exception of germ cell tumors. It is conceivable that a substantial proportion of these patients do not ever receive treatment for a subsequently diagnosed early-stage lung cancer given their competing mortality risk. For early-stage lung cancer clinical trial eligibilty, a relatively straightforward approach to addressing this clinical scenario is to exclude those patients with another 'active' cancer at the time of enrollment.

To approximate a sample more representative of a clinical trialeligible population than the overall SEER-Medicare cohort, we examined the prognostic impact of prior cancer diagnosis among the subset of early-stage lung cancer that underwent surgical resection. For these cases, all-cause survival was slightly worse among patients with a prior cancer diagnosis (HR 1.13). The magnitude of this effect is quite small and unlikely to alter clinical trial results in a meaningful way. Nevertheless, if desired, one could adjust for a history of prior cancer in a final analysis model, or even employ prior cancer diagnosis as a stratification variable. 
Importantly, this effect is less pronounced than the detrimental impact of male gender in the same models (HR 1.42, data not shown), a characteristic that is neither excluded nor stratified for in lung cancer clinical trials.

Clinicians and investigators may also have potential concerns about enrolling patients with prior cancer diagnoses due to previous exposure to certain treatments. For instance, prior chemotherapy could raise questions of both tolerability and efficacy of current systemic therapies under investigation. Given the nature of prior cancer in this population, it seems that relatively few patients would have received systemic therapy for their earlier cancer. Over $60 \%$ of prior cancer cases were in situ or localized stage. Over $50 \%$ were prostate, GI (primarily colorectal), and other GU (primarily bladder), for which systemic therapy is not routinely given for early-stage disease. Separately, eligibility criteria related to functional status and organ function may adequately address concerns about tolerance of protocol therapy. To eliminate such concerns entirely, one could exclude certain prior cancer treatments (distinct from prior cancer diagnoses) in clinical trial protocols, a practice employed in $40 \%$ of ECOG lung cancer trials (Gerber et al, 2014).

Certain limitations apply to this study. The results are not generalizable to other cancer types. The limited body of literature examining the impact of prior cancer diagnoses for other cancer types shows variable effects. Patients with lung cancer may be older, have greater smoking histories (and therefore at greater risk for multiple cancer types; Bunn and Lilenbaum, 2003; Horn et al, 2013), and have worse outcomes than patients with other cancers. SEER-Medicare data, although arguably representative of the general U.S. lung cancer population, include patients who are older and sicker than the subset of patients enrolled in clinical trials. We sought to address this shortcoming with the subset analysis of surgically resected cases which demonstrated similar survival to the overall population.

It is also possible that some of the index lung cancers diagnosed among patients with a prior cancer could represent misclassified metastases from the earlier primary tumor. However, this possibility appears unlikely given SEER's high rates of microscopic confirmation and the strict SEER algorithms and rules for coding primary cancers. Briefly, the SEER rules for classifying multiple primaries depend on the cancer site of origin, histology, diagnosis date, tumor behavior, and laterality of paired organs (Curtis et al, 2006; Johnson et al, 2014). Furthermore, contemporary pathologic and immunohistochemical techniques make this unlikely to occur in clinical practice. However, if such a bias were present, it would likely bias our results in the opposite direction of what we observed. In other words, this misclassification would result in patients with a prior cancer having an increased mortality. Therefore we are confident such misclassification is unlikely, and if present, does not explain our observed results. Finally, this study does not directly address other factors relevant to clinical trial conduct and outcomes such as tolerability of therapy. However, the proportion of patients undergoing specific treatment modalities such as surgery and radiation therapy was similar for the prior cancer and no prior cancer groups, suggesting that a prior cancer diagnosis does not inherently complicate or limit the administration of definitive lung cancer therapy.

In conclusion, prior cancer diagnosis occurs frequently among patients with early-stage lung cancer. With the rare exception of metastatic disease, a prior cancer diagnosis does not adversely impact the outcomes of these patients. Nor does a prior cancer diagnosis limit therapeutic options. In contrast to earlier studies of locally advanced and metastatic lung cancer (Gerber et al, 2014; Laccetti et al, 2016), which have outcomes substantially worse than other common cancers, the current findings in early-stage lung cancer-which has far better prognosis-suggest that the impact of prior cancer diagnoses may be comparable in other cancer types. Nevertheless, specific studies in those populations will be required.

For decades, patients with early-stage lung cancer with a history of prior cancer diagnosis have been excluded from clinical trials. Revisiting this policy and the underlying assumptions is critical. Optimal enrollment and results generalizability are key to ongoing multicenter clinical trials such as the National Cancer Institutesponsored Adjuvant Lung Cancer Enrichment Marker Identification and Sequencing Trial (ALCHEMIST), which seeks to enroll up to 8000 patients with resected non-small cell lung cancer (Gerber et al, 2015). Additionally, widespread lung cancer screening may dramatically increase the numbers of such cases diagnosed in the United States and globally, raising new clinical questions. These questions will require clinical trials that can provide real-world answers in a timely fashion.

\section{ACKNOWLEDGEMENTS}

This work was supported by the National Cancer Institute (NCI) (1R03CA191875-01A1; to DEG, SLP; EAH, LX); NCI Clinical Investigator Team Leadership Award (1P30 CA142543-01 supplement; to DEG); NCI Midcareer Investigator Award in PatientOriented Research (K24CA201543-01; to DEG); the Cancer Prevention Research Institute of Texas (CPRIT; R1208; to SLP); the Agency for Healthcare Research and Quality (1R24HS02241801; to EAH, SLP, LX) and the National Center for Advancing Translational Sciences UT Southwestern Center for Translational Medicine (U54 RFA-TR-12-006; to EAH, SLP). We would like to thank Helen Mayo, MLS, from the UT Southwestern Medical Library for assistance performing literature searches. The authors also acknowledge the efforts of the Applied Research Program, NCI; the Office of Research, Development and Information, CMS; Information Management Services (IMS), Inc.; and the Surveillance, Epidemiology, and End Results (SEER) Program tumor registries in the creation of the SEER-Medicare database. Contents of this paper are solely the responsibility of the authors and do not necessarily represent the official view of the NIH. The authors also thank Ms. Dru Gray for assistance with manuscript preparation.

\section{CONFLICT OF INTEREST}

The authors declare no conflict of interest.

\section{AUTHOR CONTRIBUTIONS}

SLP contributed to study design, statistical analysis and manuscript writing. ALL was responsible for literature searches, data interpretation and manuscript writing. LX assisted with study design and manuscript writing and performed all statistical analyses. EAH was involved with study conceptualization, data interpretation, and manuscript writing. DEG was responsible for study conceptualization/design, data interpretation, and manuscipt writing.

\section{REFERENCES}

Adamo MDL, Ruhl J (2015) National Cancer Institute SEER Coding and Staging Manual 2015. National Cancer Institute: Bethesda, MD, USA, pp 20850-29765.

Aguilo R, Macia F, Porta M, Casamitjana M, Minguella J, Novoa AM (2008) Multiple independent primary cancers do not adversely affect survival of the lung cancer patient. Eur J Cardiothoracic Surg 34(5): 1075-1080. 
Ahn DH, Mehta N, Yorio JT, Xie Y, Yan J, Gerber DE (2013) Influence of medical comorbidities on the presentation and outcomes of Stage I-III non-small-cell lung cancer. Clin Lung Cancer 14: 644-650.

Altekruse SF, Kosary CL, Krapcho M, Neyman N, Aminou R, Waldron W, Ruhl J, Howlader N, Tatalovich Z, Cho H, Mariotto A, Eisner MP, Lewis DR, Cronin K, Chen HS, Feuer EJ, Stinchcomb DG, BKe Edwards (eds) (2010) SEER Cancer Statistics Review, 1975-2007, National Cancer Institute. Bethesda, MD, http://seer.cancer.gov/csr/1975_2007/. Based on November 2009 SEER data submission, posted to the SEER web site.

Bunn Jr PA, Lilenbaum R (2003) Chemotherapy for elderly patients with advanced non-small-cell lung cancer. J Natl Cancer Inst 95(5): 341-343.

Charlson ME, Pompei P, Ales KL, MacKenzie CR (1987) A new method of classifying prognostic comorbidity in longitudinal studies: development and validation. J Chronic Dis 40(5): 373-383.

Coyte A, Morrison DS, McLoone P (2014) Second primary cancer risk-the impact of applying different definitions of multiple primaries: results from a retrospective population-based cancer registry study. BMC cancer 14: 272 .

Curtis R, Freedman D, Ron E, Ries L, Hacker D, Edwards B, Tucker M, Fraumeni JJ (2006) New malignancies among cancer survivors: SEER cancer registries. 1973-2000 Institute NC (ed) 05-5302Bethesda, MDNIH Publ.

de Moor JS, Mariotto AB, Parry C, Alfano CM, Padgett L, Kent EE, Forsythe L, Scoppa S, Hachey M, Rowland JH (2013) Cancer survivors in the United States: prevalence across the survivorship trajectory and implications for care. Cancer Epidemiol Biomarkers Prev 22(4): 561-570.

Denicoff AM, McCaskill-Stevens W, Grubbs SS, Bruinooge SS, Comis RL, Devine P, Dilts DM, Duff ME, Ford JG, Joffe S, Schapira L, Weinfurt KP, Michaels M, Raghavan D, Richmond ES, Zon R, Albrecht TL, Bookman MA, Dowlati A, Enos RA, Fouad MN, Good M, Hicks WJ, Loehrer Sr PJ, Lyss AP, Wolff SN, Wujcik DM, Meropol NJ (2013) The national cancer institute-american society of clinical oncology cancer trial accrual symposium: summary and recommendations. J Oncol Pract 9(6): 267-276.

Duchateau CS, Stokkel MP (2005) Second primary tumors involving nonsmall cell lung cancer: prevalence and its influence on survival. Chest 127(4): 1152-1158.

Friedman DR, Dubin N (1991) Case-control evaluation of breast cancer screening efficacy. Am J Epidemiol 133(10): 974-984.

Fu JB, Kau TY, Severson RK, Kalemkerian GP (2005) Lung cancer in women: analysis of the national surveillance, epidemiology, and end results database. Chest 127(3): 768-777.

Fuks A, Weijer C, Freedman B, Shapiro S, Skrutkowska M, Riaz A (1998) A study in contrasts: eligibility criteria in a twenty-year sample of NSABP and POG clinical trials. National Surgical Adjuvant Breast and Bowel Program. Pediatric Oncology Group. J Clin Epidemiol 51(2): 69-79.

Gerber D, Oxnard G, Govindan R (2015) ALCHEMIST: bringing genomic discovery and targeted therapies to early-stage lung cancer. Clin Pharmacol Ther 97(5): 447-450.

Gerber DE, Laccetti AL, Xuan L, Halm EA, Pruitt SL (2014) Impact of prior cancer on eligibility for lung cancer clinical trials. J Natl Cancer Inst 106: 11.

Goldstraw P, Crowley J, Chansky K, Giroux DJ, Groome PA, Rami-Porta R, Postmus PE, Rusch V, Sobin L (2007) The IASLC Lung Cancer Staging Project: proposals for the revision of the TNM stage groupings in the forthcoming (seventh) edition of the TNM Classification of malignant tumours. J Thorac Oncol 2(8): 706-714.

Horn L, Keedy VL, Campbell N, Garcia G, Hayes A, Spencer B, Carbone DP, Sandler A, Johnson DH (2013) Identifying barriers associated with enrollment of patients with lung cancer into clinical trials. Clin Lung Cancer 14(1): 14-18.

Iacus SM, King G, Porro G (2011) Multivariate matching methods that are monotonic imbalance bounding. J Am Stat Assoc 106(493): 345-361.

Iacus SM, King G, Porro G (2012) Causal inference without balance checking: coarsened exact matching. Polit Anal 20(1): 1-24.

Johnson C, Peace S, Adamo P, Fritz A, Percy-Laurry A, Edwards B (2014) Multiple primary and histology coding rules manual in the national cancer institute, surveillance. Epidemiology and End Results Program (Revised Edition) 2016, Bethesda, MD, USA, National Cancer Institute.

Kim ES, Bernstein D, Hilsenbeck SG, Chung CH, Dicker AP, Ersek JL, Stein S, Khuri FR, Burgess E, Hunt K, Ivy P, Bruinooge SS, Meropol N,
Schilsky RL (2015) Modernizing eligibility criteria for molecularly driven trials. J Clin Oncol 33(25): 2815-2820.

Koroukian SM, Dahman B, Copeland G, Bradley CJ (2010) The utility of the state buy-in variable in the Medicare denominator file to identify dually eligible Medicare-Medicaid beneficiaries: a validation study. Health Serv Res 45(1): 265-282.

Kris MG, Meropol NJ, Winer EP (2012) Accelerating Progress Against Cancer: ASCO's Blueprint for Transforming Clinical and Translational Cancer Research.

Laccetti AL, Pruitt SL, Xuan L, Halm EA, Gerber DE (2016) Prior cancer does not adversely affect survival in locally advanced lung cancer: a national SEERmedicare analysis. Lung Cancer (Amsterdam, Netherlands) 98: 106-113.

Laccetti AL, Pruitt SL, Xuan L, Halm EA, Gerber DE (2015) Effect of prior cancer on outcomes in advanced lung cancer: implications for clinical trial eligibility and accrual. J Natl Cancer Inst 107(4) pii: djv002.

Laccetti AL, Pruitt SL, Xuan L, Halm EA, Gerber DE (2016) Prior cancer does not adversely affect survival in locally advanced lung cancer: a national SEER-medicare analysis. Lung cancer (Amsterdam, Netherlands) 98: $106-113$.

Lara Jr PN, Higdon R, Lim N, Kwan K, Tanaka M, Lau DH, Wun T, Welborn J, Meyers FJ, Christensen S, O’Donnell R, Richman C, Scudder SA, Tuscano J, Gandara DR, Lam KS (2001) Prospective evaluation of cancer clinical trial accrual patterns: identifying potential barriers to enrollment. J Clin Oncol 19(6): 1728-1733.

Levi F, Randimbison L, Rafael BM, Manuela MC, Vecchia CL (2014) Second primary cancers in the Vaud and Neuchatel Cancer Registries. Eur J Cancer Prev 24: 150-154.

Liu YY, Chen YM, Yen SH, Tsai CM, Perng RP (2002) Multiple primary malignancies involving lung cancer-clinical characteristics and prognosis. Lung Cancer 35(2): 189-194.

Lopez-Encuentra A, Gomez de la Camara A, Rami-Porta R, Duque-Medina JL, de Nicolas JL, Sayas J. Bronchogenic Carcinoma Cooperative Group of the Spanish Society of PThoracic S (2007) Previous tumour as a prognostic factor in stage I non-small cell lung cancer. Thorax 62(5): 386-390.

Murthy VH, Krumholz HM, Gross CP (2004) Participation in cancer clinical trials: race-, sex-, and age-based disparities. JAMA 291(22): 2720-2726.

National-Cancer-Institute SEER-Medicare: Calculation of comorbidity weights (2016) SEER-Medicare Linked Database. National Cancer Institute.

Sagerup CM, Smastuen M, Johannesen TB, Helland A, Brustugun OT (2011) Sex-specific trends in lung cancer incidence and survival: a population study of 40118 cases. Thorax 66(4): 301-307.

Sankila R, Hakulinen T (1998) Survival of patients with colorectal carcinoma: effect of prior breast cancer. J Natl Cancer Inst 90(1): 63-65.

Schootman M, Jeffe DB, Lian M, Aft R, Gillanders WE (2008) Surveillance mammography and the risk of death among elderly breast cancer patients. Breast Cancer Res Treat 111(3): 489-496.

Scoggins JF, Ramsey SD (2010) A national cancer clinical trials system for the 21st century: reinvigorating the NCI Cooperative Group Program. J Natl Cancer Inst 102(17): 1371.

Siegel RL, Miller KD, Jemal A (2015) Cancer statistics, 2015. CA Cancer J Clin 65(1): 5-29.

Tong BC, Kosinski AS, Burfeind WR, Onaitis MW, Berry MF, Harpole DH, D'Amico TA (2014) Sex differences in early outcomes after lung cancer resection: Analysis of the Society of Thoracic Surgeons' General Thoracic Database. J Thorac Cardiovasc Surg 148(1): 13-18.

Warren JL, Klabunde CN, Schrag D, Bach PB, Riley GF (2002) Overview of the SEER-Medicare data: content, research applications, and generalizability to the United States elderly population. Med Care 40(8 Suppl): IV-3-18.

Warren JL, Yabroff KR, Meekins A, Topor M, Lamont EB, Brown ML (2008) Evaluation of trends in the cost of initial cancer treatment. J Natl Cancer Inst 100(12): 888-897.

Wisnivesky JP, Halm EA (2007) Sex differences in lung cancer survival: do tumors behave differently in elderly women? J Clin Oncol 25(13): $1705-1712$.

This work is published under the standard license to publish agreement. After 12 months the work will become freely available and the license terms will switch to a Creative Commons AttributionNonCommercial-Share Alike 4.0 Unported License. 